\title{
Looking for Gd neutron captures from SN $v$ with EGADS detector
}

\section{Guillaume Pronost ${ }^{* \dagger}$}

Kamioka Observatory, ICRR, University of Tokyo

E-mail: pronost@km.icrr.u-tokyo.ac.jp

EGADS (Evaluating Gadolinium's Action on Detector Systems) is the successful prototype of the Super-Kamiokande Gadolinium project (SK-Gd), which consists in dissolving $0.2 \%$ of gadolinium sulfate in the pure water of the Super-K detector. EGADS is a 200 tons tank, filled with gadolinium dopped water, and designed as a miniature version of Super-K. Gadolinium has a high neutron capture cross-section, and provides a clear signal for these neutron captures with the emission of 3-4 $\gamma_{\mathrm{s}}$ with a total energy of $\sim 8 \mathrm{MeV}$. Its presence allows to improve the separation between the $\bar{v}_{e}$ inverse $\beta$ decay interactions $\left(\bar{v}_{e}+p \rightarrow n+e^{+}\right)$producing neutrons, and the background interactions not producing neutrons. Thus increasing the efficiency of the Supernova Relic Neutrino research in the Super-K analysis, as well as the detection of future supernovae.

The EGADS research group within the Super-K collaboration now aims to use EGADS as an autonomous detector, named EGADS (Employing Gadolinium to Autonomously Detect Supernovae), for the detection of the next near-by supernovae explosions. In order to improve the sensitivity of the detector to the SNe, new electronics, based on the current Super-K IV electronics, are being installed. Moreover, the EGADS group has already collected about 2 years of data since May 2015. The analysis of this data can allow to provide a picture of the actual background of the EGADS detector for the track of the $\mathrm{SNe}$, as well as a picture of the new backgrounds we will face in the SK-Gd project.

35th International Cosmic Ray Conference

10-20 July, 2017

Bexco, Busan, Korea

\footnotetext{
* Speaker.

${ }^{\dagger}$ This work was supported by KAKENHI Grant-in-Aid for Scientific Research on Innovative Areas 26104008.
} 


\section{Introduction}

Since 1987, with the detection of the neutrino for the supernova SN1987A by Kamiokande, Baksan, and IMB, it is know that supernovae produce neutrino. Several experiments are expecting to detector the neutrinos from the next close supernova, like the Super Kamiokande experiment. In order to observe the neutrino burst from the $\mathrm{SN}$, this one needs to occurs in a short range around Earth, such as in the Milky Way galaxy. 2 or 3 of such galactic SNe are expected per century [1]. However, past and present SNe from farther away produced also neutrino burst, which should still be present in the universe forming a diffuse background. These neutrinos, called Supernova Relic Neutrinos (SRN), were up to now, never detected.

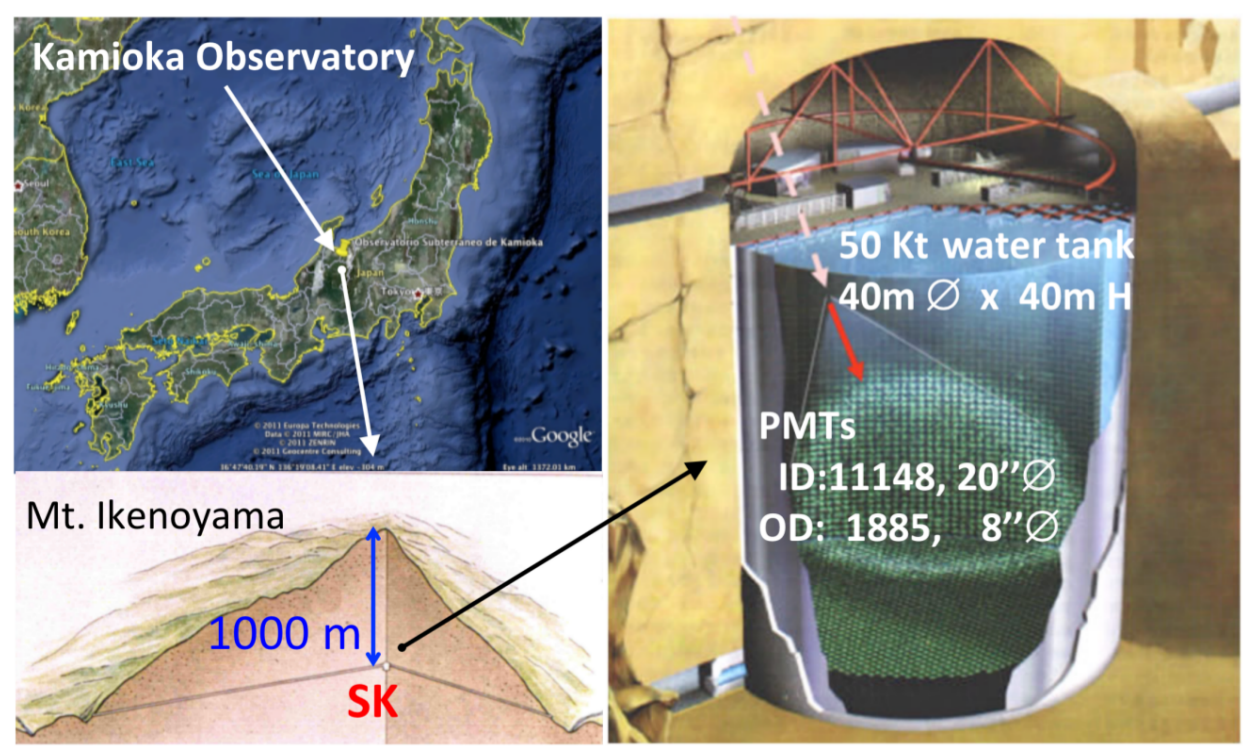

Figure 1: The Super Kamiokande experiment, located under (Mt.) Ikenoyama, in the Kamioka Observatory in the Gifu Prefecture in Japan. The 50 ktons detector is divided into an inner detector (ID, 32 kton of water) surrounded by an outer detector(OD, 18 kton).

Super Kamiokande experiment is aiming at the detection of the SRN signal, using is well known 50 ktons water Čerenkov detector in the Kamioka mine in Japan, as shown in Fig. 1. The Super-K collaboration currently holds the best limits on the SRN rate [2]. The main difficulties on the SRN detection is their low rate, compared to the high rate of other particle interactions producing similar pattern in the detector. The main component of the SRN signal is expected to be $\bar{v}_{e}$ Inverse $\beta$ Decay (IBD) interactions, due to the neutrino interaction cross-sections and the predicted SN neutrino spectrum. IBD interaction is the interaction of a $\bar{v}_{e}$ with a proton, producing a neutron and a $e^{+}$. Most of the background interactions in the SRN analysis do not produce neutron. Hence, the capability to detect the neutron production would allow to improve the signal over background ratio. In water, it is possible to detect neutron capture on hydrogen nuclei, with the detection of the $2.2 \mathrm{MeV} \gamma$ ray. However, due to the low $\mathrm{H}$ neutron capture cross section, and to the low energy of the produced $\gamma$ ray, Super-K was only able to reach a neutron detection efficiency of $17.74 \pm 0.04_{\text {stat. }} \pm 1.05_{\text {sys. }} \%$ which did not allow to improve the SRN rate limits [3]. 
In 2004, Mark Vaggins and John F. Beacom proposed to load the Super Kamiokande detector with gadolinium nuclei in order to discriminate neutron from anti-neutrino interactions [4]. Gadolinium has the highest thermal neutron cross-section among any stable nuclei (259000 barns), and produces a $8 \mathrm{MeV} \gamma$ cascade of 3-4 $\gamma_{\mathrm{s}}$ when capturing a neutron. According to studies, these two characteristic would allow to increase the neutron detection efficiency in Super-K to $\sim 80 \%$ with a loading of $0.1 \%$ of $\mathrm{Gd}$. In order to test the feasibility of this project, called SK-Gd, the Super-K collaboration build the EGADS (Evaluating Gadolinium's Action on Detector Systems) prototype in 2013.

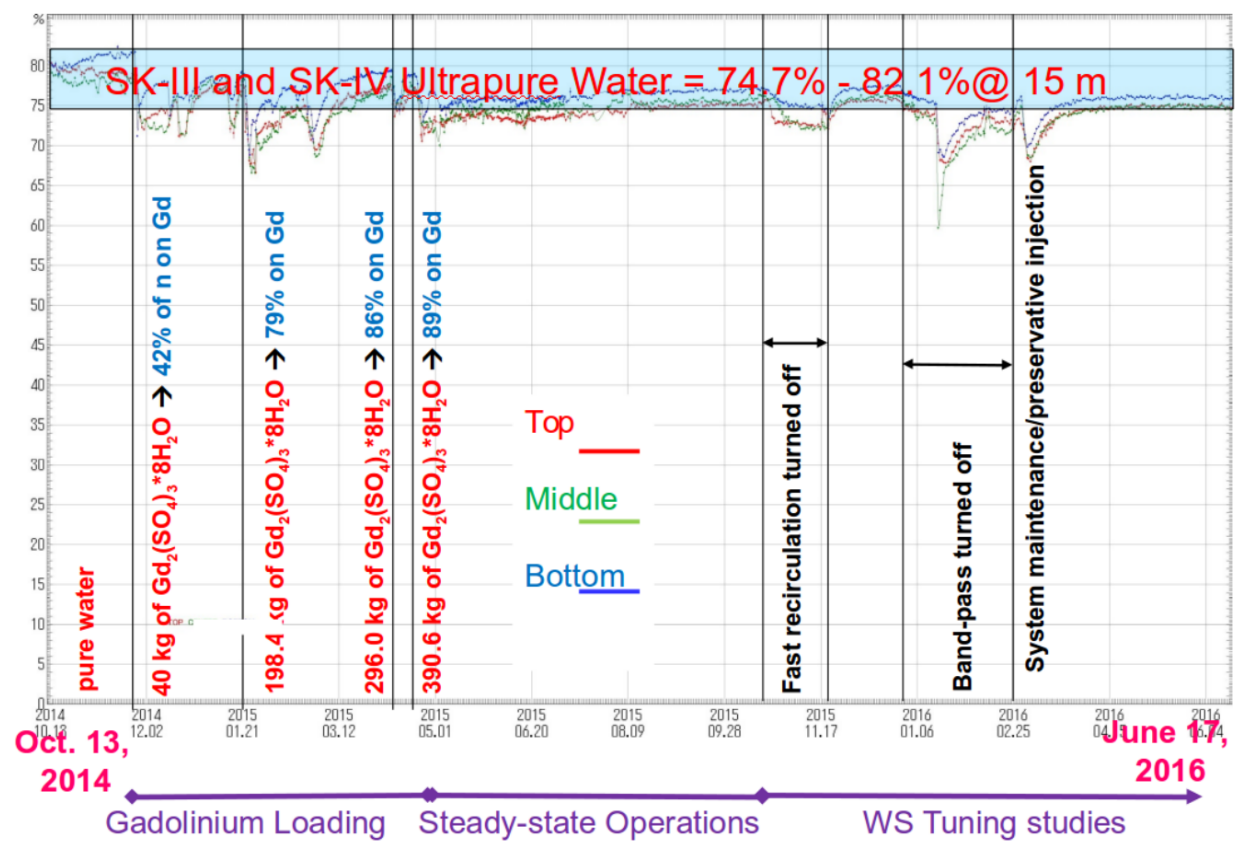

Figure 2: EGADS water transparency from October 2013 to June 2016. The blue band indicate the Super-K range of operation since its last upgrade.

EGADS is a 200 tons water Čerenkov detector, dopped with $0.2 \%$ of gadolinium sulfate (corresponding to $0.1 \%$ of gadolinium). It has been successfully running with gadolinium water for about 2 years, since May 2015. Fig. 2 shows the stability water transparency in EGADS since the gadolinium injection, which indicate a transparency at a similar level than Super-K current transparency. Thanks to this successful and stable operation, the Super Kamiokande collaboration has approved the SK-Gd project.

Now, the EGADS group of the Super Kamiokande collaboration is willing to use the EGADS detector as an autonomous supernova detector, named EGADS (Employing Gadolinium to Autonomously Detect Supernovae), in order to detect the next galactic SN. In order to reach this goal, some additional step are necessary. About 2 years of data have been collected by EGADS so far, however, up to now there were not fully analyzed. A details analysis is required in order to provide a good understanding of the backgrounds and the expected neutrino signal. In addition, the current data acquisition system is not stable enough to handle a galactic supernova burst. An upgrade of the DAQ electronics is then currently on-going. In this poster, the status of the analysis of Gd neutron capture events, as well as the status of the upgrade of the DAQ electronics, are presented. 


\section{Gd neutron capture analysis in EGADS}

In order to analyze the EGADS data properly, a good understanding of the Gd neutron capture in water is necessary. To do so, we developed a source able to mimic the IBD signal in the detector. The expected IBD signal is a delayed coincidence between a prompt signal, the energy deposition of the position as a Čerenkov emission, and a delayed signal, the neutron capture. In order to mimic this, we used a standard source of Am/Be. The combination of americium and beryllium allows to produce thermal neutron as shown in the following equation:

$$
\begin{aligned}
& { }_{95}^{241} \mathrm{Am} \rightarrow{ }_{2}^{4} \alpha+{ }_{93}^{237} \mathrm{~Np} \\
& { }_{4}^{9} \mathrm{Be}+{ }_{2}^{4} \alpha \rightarrow{ }_{6}^{12} \mathrm{C}+{ }_{0}^{1} n
\end{aligned}
$$

In addition, after its production, the ${ }_{6}^{12} 60$ has a probability of $\sim 60 \%$ (depending of the capsule) to produce a $4.44 \mathrm{MeV} \gamma$ [5]. In order to mimic a Čerenkov light, the Am/Be source is settle on top of BGO scintillator crystal, as shown in Fig. 3. The $\gamma$ is expected to interact in the BGO crystal and produce a scintillation light cascade, mimicking the Čerenkov light. However, the BGO crystal produces to much light, a black sheet with small holes is then wrapped around it in order to reduce the light intensity. .

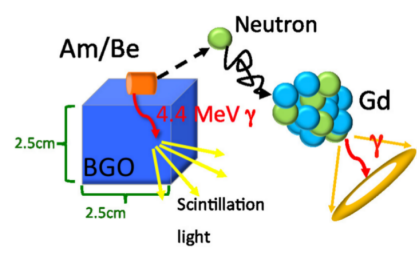

Figure 3:

The Am/Be source was deployed in EGADS detector in 3 different positions along the $\mathrm{z}$ axis: at the center of the detector, at $+1 \mathrm{~m}$, and at $-1 \mathrm{~m}$. The current DAQ electronics used hardware (HW) trigger in order to register the energy deposition in the detector. These HW triggers depends mostly on a hitsum condition, with some additional external triggers indicating the light source flashes. Two kinds of HW trigger are useful for the analysis of the Am/Be run, shown in Tab. 1, the other HW triggers are rejected from the analysis.

\begin{tabular}{|c|c|c|}
\hline HW Trigger & Hit condition & Other condition \\
\hline Prompt & 40 & - \\
Delayed & 5 & 1ms after a Prompt HW trigger \\
\hline
\end{tabular}

Table 1:

These trigger are then fitted with the BONSAI fitter from the Super-K low energy analysis. Some standard cuts on the quality of the fit are applied in the off-line analysis, as well as some additional coincidence cuts:

$$
\Delta t_{\text {Prompt-Delayed }} \in[2,500] \mu s
$$




$$
\Delta d_{\text {Source-Event }}<2 m
$$

where $\Delta t_{\text {Prompt-Delayed }}$ is the time difference between the prompt trigger and the delayed trigger, and $\Delta d_{\text {Source-Event }}$ is the distance between the source position and the event reconstructed position. The lower limit on the $\Delta t_{\text {Prompt-Delayed }}$ cut was defined to remove late light coming from the BGO crystal seen as delayed event. The $\Delta d_{\text {Source-Event }}$ was defined to remove the accidental background coming from outside and from the PMTs. Fig. 4 shows the distribution of time differences between prompt and delayed events after application of these cuts. The exponential fit of the distribution indicate a characteristic of $31.32 \pm 0.76$ (stat.) $\mu \mathrm{s}$, which is consistent with the expectation for $0.1 \% \mathrm{Gd}$ concentration. The flat component of the fit function indicate the BG rate in the sample.

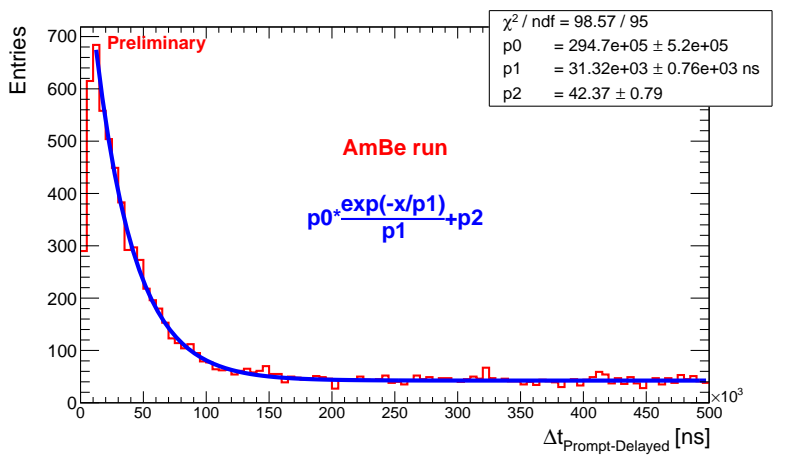

Figure 4: Distribution of the difference of time between prompt and delayed events in $\mathrm{Am} / \mathrm{Be}$ run (red) with $\mathrm{Am} / \mathrm{Be}$ source deployed in the center of the detector. The blue line indicates the fit of the distribution with an exponential + flat component function.

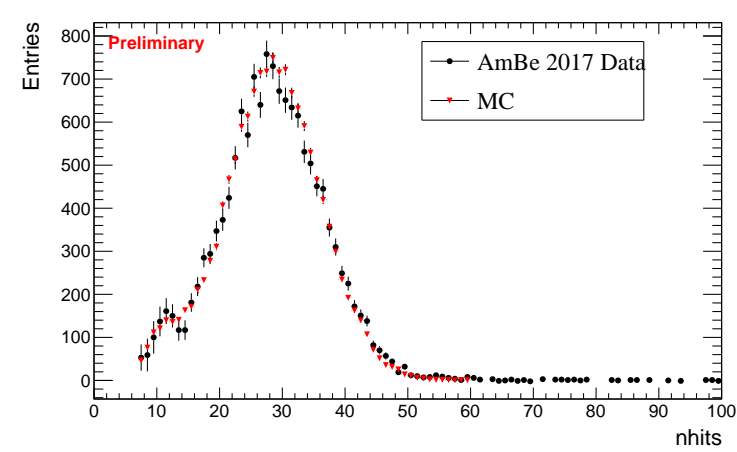

Figure 5: Distribution of the number of PMT hits for $\mathrm{Am} / \mathrm{Be}$ data (black) and $\mathrm{MC}$ (red) with $\mathrm{Am} / \mathrm{Be}$ source deployed in the center of the detector. Statistical background subtraction was applied on the data. Plot from [6].

In order to sub-sequentially removed the remaining background (BG), a statistical background subtraction methods was applied. This method consists in the use of the sample of delayed events rejected by the $\Delta t_{\text {Prompt-Delayed }}<500 \mu$ s cut as a background sample. Fig. 5 shows the spectrum of the number of hits from calibration run with $\mathrm{Am} / \mathrm{Be}$ source deployed in the center of the detector, after application of the statistical background subtraction. The same distribution from a GEANT4 MC simulation is shown. The efficiency of the Gd neutron capture selection was measured to be $85.3 \pm 0.9 \%$ (stat) in data and $84.4 \pm 0.3 \%$ (stat) in MC. This result indicates a good agreement between data and MC.

The analysis of 2 years of data from EGADS detector will allow us to have a better understanding of the BGs in order to develop a SN alarm system. In order to achieve it, the development of analysis tools and BG studies are currently on-going.

\section{Electronics upgrade}

The EGADS DAQ electronics was based on the Analog Timing Module technology (ATM). It was using old ATM modules used in Super-K before the last electronics upgrade in 2008. The 
ATM electronics registers events with a $1.3 \mu$ s event window and at a $3 \mathrm{kHz}$ rate. It also uses a $\mathrm{HW}$ hitsum trigger, as shown previously in Tab. 1. This lead to an important dead time. In addition, the ATM electronics was seen to be instable during calibration periods when high rate $(>100 \mathrm{~Hz})$ source were deployed in the detector. In case of a close supernova an event rate of several hundred of $\mathrm{Hz}$ can be expected in EGADS detector (up to several $\mathrm{kHz}$ for very close supernova). The ATM electronics was then not expected to be able to handle such major event.

The EGADS group decided to upgrade the electronics of the detector with more efficient technology. We decided to use the electronics used in Super-K since 2008, called QBEE (QTCBased Electronics with Ethernet). These modules, shown in Fig. 6, use high-speed charge-to-time converter IWATSU CLC1101, developed by the Super-K collaboration and IWATSU company [7]. These custom circuits allow to apply individual threshold to each PMTs, as well as high rate signal processing ( $>1 \mathrm{MHz}$ ) with 3 channels. The QBEE modules integrate also multi-hit time to digital converters and field-programmable gate arrays. They receive a $60 \mathrm{MHz}$ clock signal and allow an external readout via Ethernet connection.

The QBEE DAQ system uses a periodic trigger which reads $\sim 17 \mu$ s blocks, where all PMT hits are register, with a clock rate of $60 \mathrm{kHz}$. This allows to have virtually no dead time. In addition, with QBEE DAQ system, we can by-pass the HW trigger logic, and directly use a software (SW) trigger. This SW trigger allows to have variable event window, depending of the physics interactions we are looking for. This brings more flexibility on the trigger selection.

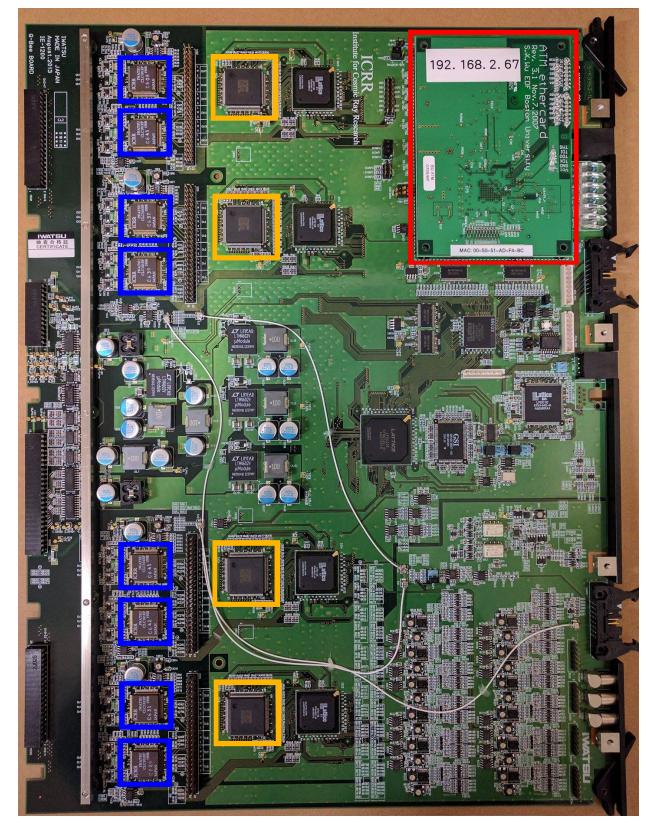

Figure 6: View of a QBEE module from EGADS electronics. The blue squares indicate the position of the custom QTC circuits, the yellow squares indicate the position of the TDC circuits, and the red square indicates the position of the Ethernet daughter board of the QBEE module.

On the 5th of July 2017, the ATM DAQ electronics of EGADS was stopped, and the installation of the QBEE DAQ electronics started. Currently, the QBEE DAQ electronics is running fine since several weeks, but not yet in its final stage. EGADS has 4 kinds of PMTs, during the first weeks of the installation we focused on tunning the DAQ for the Super-K PMTs which represent 
$\sim 94 \%$ of the PMTs in EGADS tank. The threshold for these PMTs was tunned to keep a dark noise below the averaged dark noise with ATM electronics, and to minimize the high rate PMTs. Fig. 7 shows the mean dark noise from standard PMT with QBEE electronics as function of the threshold applied. The dark noise rate is equal to the averaged dark noise with ATMs for a hit threshold of $-0.59 \mathrm{mV}$. However, the number of noisy PMTs (with dark noise $>10 \mathrm{kHz}$ ) start to increase when the hit threshold is set above $-0.63 \mathrm{mV}$. Therefore, we determined the hit threshold for Super-K PMT to be $-0.63 \mathrm{mV}$.

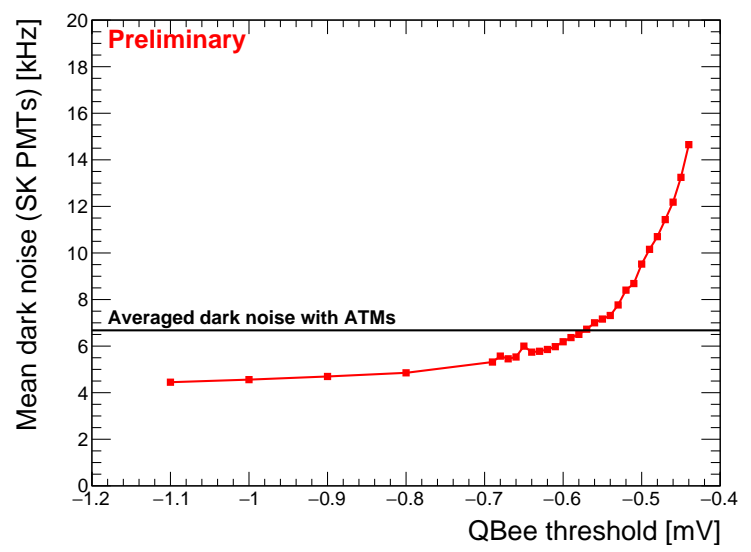

Figure 7: Mean dark noise for Super-K PMTs in EGADS tank, with QBEE electronics, as function of the hit threshold. The dotted line indicated the averaged dark noise for standard PMTs with the ATM electronics.

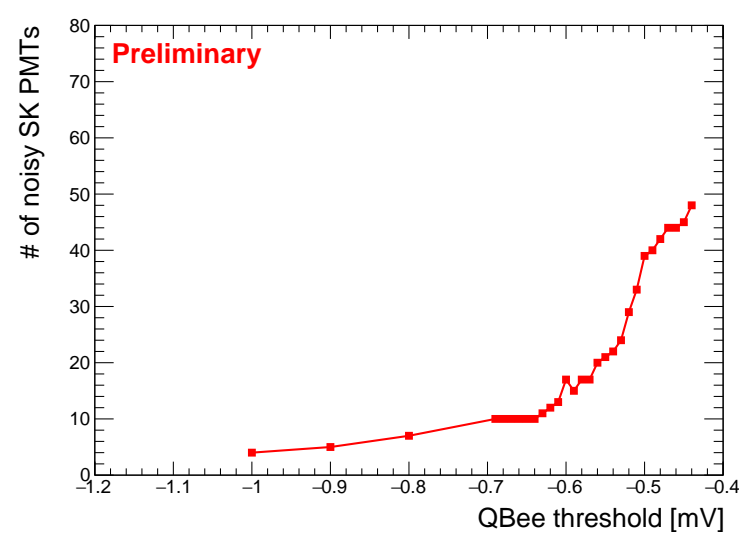

Figure 8: Number of noisy PMTs among Super-K PMTs in EGADS tank, with QBEE electronics, as function of the hit threshold.

Similar studies are on-going for the three other types of PMTs we have in EGADS tank. In addition, the tunning of the SW trigger condition is also on-going. The final step of this electronics upgrade will be to reproduce the results from the calibration campaign we did before the QBEE installation. We expect to have the final setup and configuration running this July.

\section{Summary}

The transition of EGADS from a Super-K prototype to an actual SNe neutrino detector is on-going. The analysis of the physics data would be an important step in order to understand the backgrounds and the different neutrino signals we should have register. The upgrade of the DAQ electronics will also us to have a stable detector able to handle the burst of a supernova. Several additional supernova analysis are foreseen, and will be allowed thanks to the capabilities of the QBEE electronics. In order to be able to properly detect SNe, two remaining work will be conducted at the end of the electronics upgrade. A GPS fiber is going to be deployed from the Super-Kamiokande GPS system to EGADS electronics hut. An online SN alert system is going to be developed in order to provide us the ability to immediately detect a SN event, in the goal to warn the international community soon after this detection.

At the same time, several works on the improvement of the Gd sulfate radio-purity are ongoing in the EGADS group for SK-Gd, as present in this conference in [8]. It is foreseen to drain 
the EGADS tank in the next months in order to loaded it with a new Gd sulfate with a better radio-purity. This is expected to decreases the accidental BG in EGADS tank.

Once this will be done, the EGADS detector can be expected to be running stably. We hope to able to detect the next close SN.

\section{References}

[1] E. Cappellaro, R. Evans, and M. Turatto, Astron. Astrophys. 351, 459 (1999).

[2] K. Bays et al. (Super Kamiokande collaboration), Supernova Relic Neutrino Search at Super-Kamiokande, Phys. Rev. D85 (2012) 052007

[3] H. Zhang et al. (Super Kamiokande collaboration), Supernova Relic Neutrino Search with Neutron Tagging at Super-Kamiokande IV, Astroparticle Phys. 60 (2014) 41

[4] John F. Beacom and Mark R. Vagins, Antineutrino Spectroscopy with Large Water C̆erenkov Detectors, Phys. Rev. Lett. 93, 171101 (2004)

[5] J. Scherzinger et al., Tagging fast neutrons from an 241Am/9Be source, Applied Radiation and Isotopes 98 (2015) 74

[6] M. Murdoch, EGSIM update, Internal Communication in Super-Kamiokande collaboration meeting (Oct. 2017)

[7] H. Nishino et al., High-speed charge-to-time converter ASIC for the Super-Kamiokande detector, Nucl.Instrum.Meth.A610:710-717,2009

[8] S. Ito for the Super-Kamiokande collaboration, Status of RI background reduction for SK-Gd, Poster presented in ICRC 2017 